Alma Mater Studiorum - Università di Bologna DEPARTMENT OF ECONOMICS

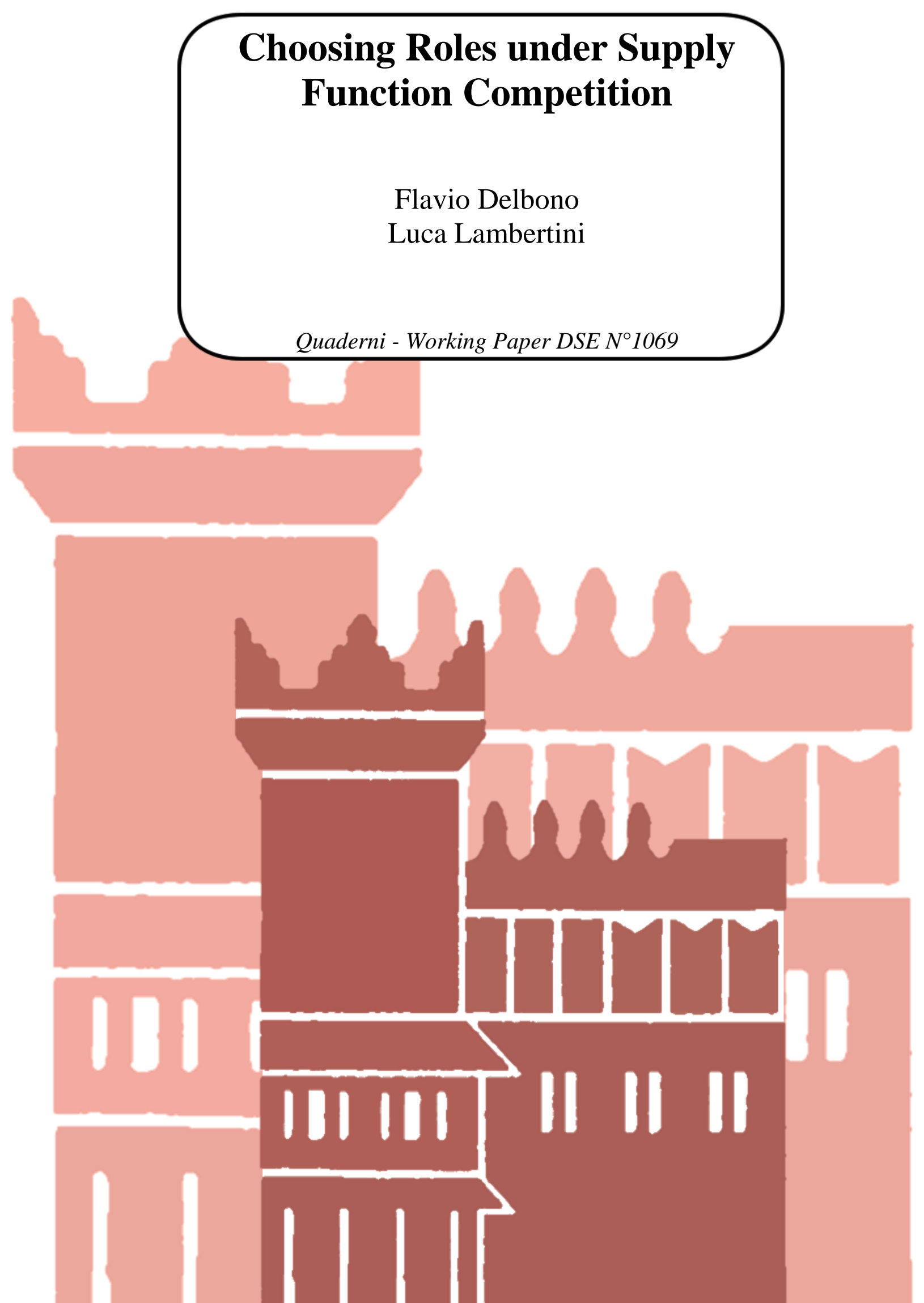




\title{
Choosing Roles under Supply Function Competition
}

\author{
Flavio Delbono ${ }^{\#}$ and Luca Lambertini ${ }^{\S}$ \\ \# Department of Economics, University of Bologna \\ Piazza Scaravilli 2, 40126 Bologna, Italy \\ flavio.delbono@unibo.it \\ $\S$ Department of Economics, University of Bologna \\ Strada Maggiore 45, 40125 Bologna, Italy \\ luca.lambertini@unibo.it
}

June 7, 2016 


\begin{abstract}
We investigate an extended game with observable delay under duopolistic competition in affine supply functions. Firms use the intercepts of supply functions as their strategic variables. Best replies are downward (upward) sloping if the common slope of supply functions is sufficiently low (high). Accordingly, simultaneous (sequential) play is selected at the subgame perfect equilibrium when best replies are negatively (positively) sloped. There exists a unique value of the slope at which best replies are orthogonal and the choice between simultaneous and sequential play is immaterial.
\end{abstract}

JEL Codes: D43, L13

Keywords: supply function; strategic complements; strategic substitutes; endogenous timing 


\section{Introduction}

The main question addressed in this paper deals with the leader-follower choice under (affine) supply function competition within an extended game with observable delay à la Hamilton and Slutsky (1990). To this end, we model supply functions as in Menezes and Quiggin (2012), where firms choose the intercepts of affine supply functions. Doing so, Menezes and Quiggin (2012) prove the existence of a continuum of equilibria ranging from Cournot (when the slope of supply functions is nil) to Bertrand (when the slope tends to infinity).

In a widely cited paper, Bulow et al. (1985) introduce an important criterion to classify the strategic nature of different choice variables in oligopolistic games. Borrowing a well established terminology from demand theory, they identify a binary taxonomy opposing strategic complements to strategic substitutes.

We first show that the (intercept of) supply function may feature both strategic substitutability or complementarity, depending on the steepness of supply functions. More precisely, we identify a unique critical threshold of the slope, below which competition takes place in strategic substitutes, whereas the opposite applies above the threshold. The switch between strategic substitutability and complementarity does not arise in the initial modelization of linear supply functions dating back to Klemperer and Meyer (1989) where the intercept is zero. In such a setting firms compete in the space of slopes, and it turns out that slopes are strategic complements and the resulting Nash equilibrium in supply functions - which is unique in absence of uncertainty lies between Cournot and Bertrand. The first author pointing out the emergence of strategic complementarity among slopes is Laussel (1992), and the 
same property is stressed again in Akgün (2004). ${ }^{1}$

Solving the extended game with observable delay, we prove that when supply functions are not too steep, firms play simultaneously as soon as possible because competition takes place in strategic substitutes, while they play sequentially (and therefore the mixed strategy equilibrium is also relevant) when supply functions are sufficiently steep and the game is in strategic complements. In correspondence of the critical threshold of the slope, best replies are orthogonal and Stackelberg and Nash equilibria coincide.

The paper is organized as follows. In the next section we study the properties of affine supply functions when firms choose intercepts in a well known model of duopoly with product differentiation. The extended game with observable delay and its equilibrium analysis, including the mixed strategy equilibrium, are illustrated in section 3. Section 4 concludes.

\section{The model}

We consider the model of differentiated duopoly introduced by Spence (1976) and Singh and Vives (1984). The utility function of the representative consumer is

$$
U=a\left(q_{1}+q_{2}\right)-\frac{1}{2}\left(q_{1}^{2}+q_{2}^{2}+2 \sigma q_{1} q_{2}\right)
$$

where $q_{i}$ is the quantity of the variety supplied by firm $i=1,2, a>0$ and parameter $\sigma \in(0,1]$ measures the degree of product substitutability, i.e., $\sigma$ is an inverse measure of product differentiation. When $\sigma=1$, the product is

\footnotetext{
${ }^{1}$ Ciarreta and Gutierrez-Hita (2006) model linear supply functions in a supergame where the focus is on implicit collusion. Since they set intercepts to zero and firms choose slopes, their game is one in strategic complements. Vives (2011) follows the same approach as in Klemperer and Meyer (1989) using the notion of residual demand curve under market clearing and leaving aside the issue of the strategic nature of supply functions.
} 
homogeneous. ${ }^{2}$ The direct demand functions resulting from the constrained maximisation problem are:

$$
q_{i}=\max \left\{0, \frac{a}{1+\sigma}-\frac{p_{i}}{1-\sigma^{2}}+\frac{\sigma p_{j}}{1-\sigma^{2}}\right\}, i=1,2 .
$$

System (2) can be inverted to yield the direct demand system:

$$
p_{i}=a-q_{i}-\sigma q_{j}, i=1,2
$$

On the supply side, both single-product firms operate with the linear cost function $C_{i}=c q_{i}$, with $c \in(0, a)$. The profit function of firm $i$ is

$$
\pi_{i}=\left(p_{i}-c\right) q_{i}
$$

Before delving into the analysis of supply function competition, it is worth recalling two basic properties of Cournot and Bertrand competition. Define the best reply of firm $i$ in the quantity and price spaces, respectively, as $q_{i}^{*}\left(q_{j}\right)$ and $p_{i}^{*}\left(p_{j}\right)$. Their slopes are

$$
\frac{\partial q_{i}^{*}\left(q_{j}\right)}{\partial q_{j}}=-\frac{\sigma}{2}<0 \forall \sigma \in(0,1]
$$

and

$$
\frac{\partial p_{i}^{*}\left(p_{j}\right)}{\partial p_{j}}=\frac{\sigma}{2}>0 \forall \sigma \in(0,1]
$$

as we know from Singh and Vives (1984). On the basis of (5-6), one establishes that, for any degree of product substitutability, best replies are decreasing in the quantity space and increasing in the price space. In the jargon of Bulow et al. (1985), this amounts to saying that quantities are strategic substitutes and prices are strategic complements when goods are substitutes in demand.

\footnotetext{
${ }^{2}$ If $\sigma=0$, the two varieties do not interact and firms are independent monopolists. We also disregard the range $\sigma \in[-1,0)$, where products are complements.
} 
To model competition in supply functions, we focus on linear supply functions. More precisely, we adopt the formulation introduced by Menezes and Quiggin (2012). The supply function of firm $i$ is defined as $S_{i}=\alpha_{i}-$ $c / 2+\beta_{i}\left(p_{i}-c\right)$, and the ex ante market clearing condition is $S_{i}=q_{i}$, where $q_{i}$ is defined as in (2). The presence of two varieties requires imposing two market-clearing conditions. Taking the individual demand function in (2), whenever $q_{i}>0$, market clearing requires imposing:

$$
\alpha_{i}-\frac{c}{2}+\beta_{i}\left(p_{i}-c\right)=\frac{a}{1+\sigma}-\frac{p_{i}}{1-\sigma^{2}}+\frac{\sigma p_{j}}{1-\sigma^{2}}
$$

Solving system (7) w.r.t. $p_{i}$ and $p_{j}$, and substituting the resulting marketclearing prices into the supply functions, we can write firm $i$ 's supply function as

$$
\begin{gathered}
S_{i}=\frac{2\left[\alpha_{i}\left(1+\beta_{j}\right)+\beta_{i}\left(a\left(1+(1-\sigma) \beta_{j}\right)-\sigma \alpha_{j}\right)\right]}{2\left[\beta_{i}\left(1+\beta_{j}\left(1-\sigma^{2}\right)\right)+1+\beta_{j}\right]}- \\
\frac{c\left[1+\beta_{j}+\beta_{i}\left(2-\sigma+2 \beta_{j}(1-\sigma)\right)\right]}{2\left[\beta_{i}\left(1+\beta_{j}\left(1-\sigma^{2}\right)\right)+1+\beta_{j}\right]}
\end{gathered}
$$

and the profit function of firm $i$ therefore writes as

$$
\pi_{i}=\left(a-S_{i}-\sigma S_{j}-c\right) S_{i}
$$

As in Menezes and Quiggin (2012), we assume that competition in supply function takes place in the space of intercepts $\left(\alpha_{1}, \alpha_{2}\right)$, with $\beta_{1}=\beta_{2}=$ $\beta \in[0, \infty)$. The advantage of using the approach of Menezes and Quiggin (2012) lies in the fact that it generates a continuum of equilibria ranging from Cournot (when $\beta=0$ ) to Bertrand, which is reached in the limit as $\beta$ tends to infinity.

Taking the first order condition (FOC) for the maximisation of (9) w.r.t. 
$\alpha_{i}$ and solving, one obtains the best reply function of firm $i:^{3}$

$$
\begin{aligned}
\alpha_{i}^{*}\left(\alpha_{j}\right)= & \frac{2 a[1+(1-\sigma) \beta]\left[1-\beta^{2}\left(1-\sigma^{2}\right)\right]-2 \sigma \alpha_{j}\left[1-\beta^{2}\left(1-\sigma^{2}\right)\right]}{4(1+\beta)\left[1+\beta\left(1-\sigma^{2}\right)\right]}+ \\
& \frac{c[1+\beta(1-\sigma)][\sigma+\beta(1+\sigma)(2-\sigma+2 \beta(1-\sigma))]}{4(1+\beta)\left[1+\beta\left(1-\sigma^{2}\right)\right]}
\end{aligned}
$$

To understand the strategic nature of competition in supply functions, it suffices to take the partial derivative of $\alpha_{i}^{*}\left(\alpha_{j}\right)$ w.r.t. $\alpha_{j}$ :

$$
\frac{\partial \alpha_{i}^{*}\left(\alpha_{j}\right)}{\partial \alpha_{j}}=-\frac{\sigma\left[1-\beta^{2}\left(1-\sigma^{2}\right)\right]}{2(1+\beta)\left[1+\beta\left(1-\sigma^{2}\right)\right]}
$$

Inspecting (11), which is continuous and twice differentiable in $\beta$, for all $\beta \in[0, \infty)$, one proves the following:

Lemma $1 \partial \alpha_{i}^{*}\left(\alpha_{j}\right) / \partial \alpha_{j} \lesseqgtr 0$ for all $\beta \lesseqgtr \widehat{\beta} \equiv 1 / \sqrt{1-\sigma^{2}}$. Moreover, in $\beta=0$,

$$
\frac{\partial \alpha_{i}^{*}\left(\alpha_{j}\right)}{\partial \alpha_{j}}=-\frac{\sigma}{2}=\frac{\partial q_{i}^{*}\left(q_{j}\right)}{\partial q_{j}}
$$

and

$$
\lim _{\beta \rightarrow \infty} \frac{\partial \alpha_{i}^{*}\left(\alpha_{j}\right)}{\partial \alpha_{j}}=\frac{\sigma}{2}=\frac{\partial p_{i}^{*}\left(q_{j}\right)}{\partial p_{j}} .
$$

The Lemma shows that the individual firm's best reply derived under supply function competition delivers strategic substitutability (complementarity) as under Cournot (Bertrand) behaviour in the inf (sup) of the support of parameter $\beta$. Additionally, strategic substitutability holds for all $\beta \in\left[0,1 / \sqrt{1-\sigma^{2}}\right)$ while strategic complementarity emerges for all $\beta \in$ $\left(1 / \sqrt{1-\sigma^{2}}, \infty\right)$. In correspondence of $\beta=1 / \sqrt{1-\sigma^{2}}$ best replies are orthogonal in the space $\left(\alpha_{1}, \alpha_{2}\right)$. The intuition is fairly clear: as product differentiation tends to vanish (i.e., $\sigma$ approaches one), we enter a domain in

\footnotetext{
${ }^{3}$ The second order condition, which is omitted for brevity, is satisfied in correspondence of $\alpha_{i}=\alpha_{i}^{*}\left(\alpha_{j}\right)$.
} 
which firms' best replies are upward sloping, and therefore they operate under strategic complementarity mimicking price competition which is reached in the limit as $\beta$ goes to infinity.

The symmetric Nash equilibrium is attained at

$$
\alpha^{N}=\frac{2 a\left[1-\left(1-\sigma^{2}\right) \beta^{2}\right]+c\left[(1+\beta)(2 \beta+\sigma)-\beta(1+2 \beta) \sigma^{2}\right]}{2(1+\beta)(2+\sigma)-2 \beta \sigma^{2}}
$$

delivering profits

$$
\pi^{N}=\frac{(a-c)^{2}(1+\beta)\left[1+\left(1-\sigma^{2}\right) \beta\right]}{[2+\sigma+(2-\sigma)(1+\sigma) \beta]^{2}}
$$

The analysis of simultaneous play, which Menezes and Quiggin (2012) carry out under product homogeneity, shows that if supply functions are flat, the supply function Nash equilibrium collapses onto the Cournot-Nash equilibrium, while if supply functions are infinitely steep, the equilibrium degenerates into the Bertrand-Nash one. The same applies here under product differentiation. This can be verified on the basis of (13):

$$
\lim _{\beta \rightarrow 0} \pi^{N}=\frac{(a-c)^{2}}{(2+\sigma)^{2}}
$$

which is the individual Cournot-Nash profit, and

$$
\lim _{\beta \rightarrow \infty} \pi^{N}=\frac{(a-c)^{2}(1-\sigma)}{(1+\sigma)(2-\sigma)^{2}}
$$

which is the individual Bertrand-Nash profit as in Singh and Vives (1984).

This property allows Menezes and Quiggin (2012) to claim that supply function competition yields a continuum of equilibria generated by $\beta$, with the classical Bertrand and Cournot equilibria as limit cases. What we are about to prove the existence of multiple equilibria relying on Lemma 1, as the multiplicity is the consequence of the link between the slope of the supply function and that of the best reply in the $\alpha$ space. 


\section{The extended game with observable delay}

The choice between simultaneous and sequential play in a noncooperative variable sum game goes back at least to d'Aspremont and Gérard-Varet (1980). Then, from the mid-1980s, this issue has entered oligopoly theory, thanks to Gal-Or (1985), Dowrick (1986), Boyer and Moreaux (1987) and Robson (1990), inter alia. A milestone of this literature is the concept of extended game with observable delay, introduced by Hamilton and Slutsky (1990). Their framework can be summarised as follows. The market subgame is preceded by a pre-play stage in discrete strategies where firms (under complete, symmetric and imperfect information) noncooperatively select the timing of their respective moves which will be relevant in the ensuing continuous strategy stage. If simultaneous play is announced in the first stage, firms play the Nash equilibrium at the market stage. If instead sequential play is announced, multiple Stackelberg equilibria in pure strategies will exists at the market stage, together with the mixed strategy one. No time discounting is involved between stages, as time is a logical rather than chronological dimension. The extended game with observable delay allows to determine whether a game is Stackelberg solvable in the sense of d'Aspremont and Gérard-Varet (1980). Indeed, from both d'Aspremont and Gérard-Varet (1980) and Hamilton and Slutsky (1990), there emerges that sequential play is selected if and only if the Stackelberg outcome Pareto dominates the Nash one.

Using our notation, the formal structure of the extended game with observable delay can be summarised as follows. Define as $\Gamma=(N, X, \Pi)$ the two-stage extended game with observable delay. The set of players (or firms) is $N=\{i, j\}$, and $\phi_{i}\left(\alpha_{i}, \alpha_{j}\right)$ and $\phi_{j}\left(\alpha_{i}, \alpha_{j}\right)$ are the compact and convex intervals of $\mathbb{R}$ representing the actions available to agents $i$ and $j$ in the 
downstream market stage, conditional upon the choices made in the upstream stage. $\Pi$ is the payoff function vector, such that individual payoffs are defined as $\pi_{i}\left(\alpha_{i}, \alpha_{j}\right): \phi_{i}\left(\alpha_{i}, \alpha_{j}\right) \times \phi_{j}\left(\alpha_{i}, \alpha_{j}\right) \rightarrow \mathbb{R}$ and $\pi_{j}\left(\alpha_{i}, \alpha_{j}\right)$ : $\phi_{j}\left(\alpha_{i}, \alpha_{j}\right) \times \phi_{i}\left(\alpha_{i}, \alpha_{j}\right) \rightarrow \mathbb{R}$. The set of times at which firms can choose to move is $T=\left\{t_{1}, t_{2}\right\}$, i.e., early or late. The set of strategies for player $i$ is $x_{i}=T \times \Phi_{i}$, where $\Phi_{i}$ maps $T \times \phi_{j}\left(\alpha_{i}, \alpha_{j}\right)$ into $\phi_{i}\left(\alpha_{i}, \alpha_{j}\right)$.

It is worth stressing that the notion of time underpinning the extended game with observable delay is purely logical and involves no discounting. The resulting two-stage game is fully noncooperative, and the outcome of the first stage (i.e., the choice of timing) is observable to all firms alike before the second stage takes place. If in the market subgame both firms choose to move at the same time $\left(t_{i}=t_{j}\right)$, they obtain the profits associated with the simultaneous Nash equilibrium, otherwise they get the profits associated with the Stackelberg equilibrium, with $i$ moving first and $j$ moving second, or vice versa.

To characterise the Stackelberg equilibrium in supply functions, we denote the leader and the follower by $L$ and $F$, respectively. Firm $L$ maximises profits under the constraint posed by the follower's best reply function (10). The resulting equilibrium strategy for the leader is

$$
\alpha^{L}=\frac{2 a[2-\sigma+(2-\sigma(1-\sigma)) \beta]\left[1-\left(1-\sigma^{2}\right) \beta^{2}\right]+c \Psi}{2\left[2+\left(2-\sigma^{2}\right) \beta\right]\left[2-\sigma^{2}+2\left(1-\sigma^{2}\right) \beta\right]}
$$

where

$$
\begin{gathered}
\Psi \equiv 2(1-\sigma) \sigma+\left[4+\sigma\left(2-\sigma\left(6-\sigma^{2}\right)\right)\right] \beta+ \\
2\left(1-\sigma^{2}\right)[4-\sigma(1+\sigma)] \beta^{2}+2\left(1-\sigma^{2}\right)(1+\sigma)(2+\sigma) \beta^{2}>0
\end{gathered}
$$

and

$$
\alpha^{L}-\alpha^{F}=\frac{(a-c) \sigma^{2}[1+(1-\sigma) \beta]\left[1-\left(1-\sigma^{2}\right) \beta^{2}\right]}{2\left[2+\left(2-\sigma^{2}\right) \beta\right]\left[2-\sigma^{2}+2\left(1-\sigma^{2}\right) \beta\right]}
$$


which is positive for all $\beta \in\left[0,1 / \sqrt{1-\sigma^{2}}\right)$, while the opposite applies in the remainder of the admissible range of $\beta$. That is, $\alpha^{L}>\alpha^{F}$ when firms' choices are strategic substitutes, whereas $\alpha^{L}<\alpha^{F}$ under strategic complementarity.

In the special case in which $\beta=1 / \sqrt{1-\sigma^{2}}$, since best replies are orthogonal, Nash and Stackelberg equilibria coincide, in such a way that the tangency point between firm $i$ 's highest isoprofit curve and firm $j$ 's best reply function occurs in correspondence of the intersection of best replies themselves (see Figure 1).

Figure 1

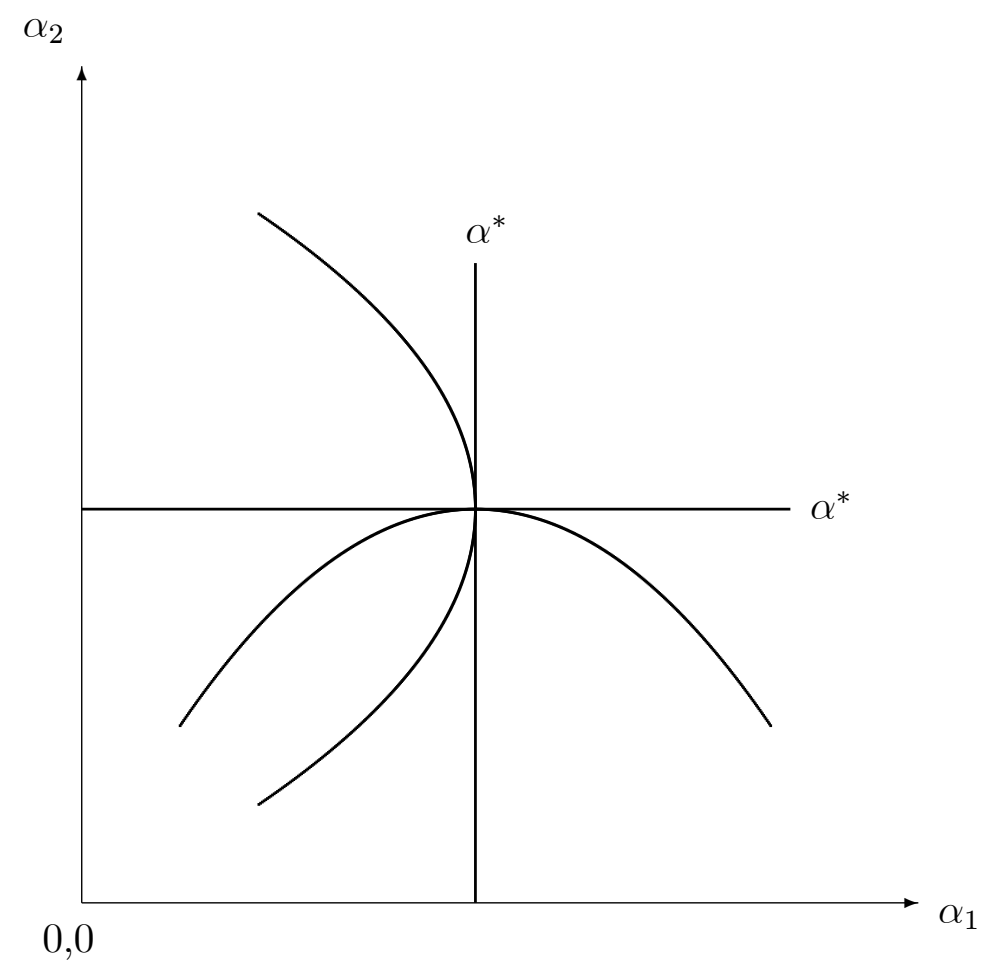

The profits generated by the Stackelberg equilibrium values of $\alpha$ 's are the 
following:

$$
\begin{gathered}
\pi^{L}=\frac{(a-c)^{2}[2-\sigma+(2-\sigma(1-\sigma)) \beta]^{2}}{4\left[2+\left(2-\sigma^{2}\right) \beta\right]\left[2-\sigma^{2}+2\left(1-\sigma^{2}\right) \beta\right]} \\
\pi^{F}=\frac{(a-c)^{2}(1+\beta)\left[1+\left(1-\sigma^{2}\right) \beta\right][4(1+\beta)-\sigma(2+\sigma(3-\sigma)) \beta-\sigma(2+\sigma)]}{4\left[2+\left(2-\sigma^{2}\right) \beta\right]\left[2-\sigma^{2}+2\left(1-\sigma^{2}\right) \beta\right]}
\end{gathered}
$$

We are now in a position to describe the pre-play stage for the choice of

\begin{tabular}{|c|c|c|}
\hline & \multicolumn{2}{|c|}{$j$} \\
\hline & $t_{1}$ & $t_{2}$ \\
\hline$i \quad t_{1}$ & $\pi^{N}, \pi^{N}$ & $\pi^{L}, \pi^{F}$ \\
\hline$t_{2}$ & $\pi^{F}, \pi^{L}$ & $\pi^{N}, \pi^{N}$ \\
\hline
\end{tabular}
timing. The resulting reduced form describing the first stage is in Matrix 1.

Matrix 1

Using expressions (13) and (19-20), we can rank the profits appearing in Matrix 1:

$$
\begin{aligned}
& \pi^{N}-\pi^{F}=\Lambda(\beta, \sigma)(a-c)^{2}\left[1-\left(1-\sigma^{2}\right) \beta^{2}\right] \\
& \pi^{L}-\pi^{N}=\Xi(\beta, \sigma)(a-c)^{2}\left[1-\left(1-\sigma^{2}\right) \beta^{2}\right]^{2} \\
& \pi^{L}-\pi^{F}=\Omega(\beta, \sigma)(a-c)^{2}\left[1-\left(1-\sigma^{2}\right) \beta^{2}\right]
\end{aligned}
$$

where $\Lambda(\cdot), \Xi(\cdot)$ and $\Omega(\cdot)$ are positive polynomials. While $\pi^{L} \geq \pi^{N}$ always, being nil at $\beta=1 / \sqrt{1-\sigma^{2}},(21)$ and (23) are positive for all $\beta \in$ $\left(0,1 / \sqrt{1-\sigma^{2}}\right)$ and negative for all $\beta>1 / \sqrt{1-\sigma^{2}}$. This, joint with Lemma 1, proves:

Proposition 2 For all $\beta \in\left[0,1 / \sqrt{1-\sigma^{2}}\right),\left(t_{1}, t_{1}\right)$ is the unique Nash equilibrium at the first stage of the extended game with observable delay, generated 
by strictly dominant strategies. For all $\beta>1 / \sqrt{1-\sigma^{2}}$, the first stage produces two pure-strategy Nash equilibria involving sequential play along the secondary diagonal.

Since for low values of $\beta$ best replies are decreasing, or, equivalently, firms are competing in strategic substitutes, there exists a strict incentive to choose $t_{1}$ in order to avoid the follower's role. As a result, firms play simultaneously because the game replicates a basic feature of the Cournot model. Conversely, if $\beta$ is large enough, competition takes place in strategic complements and the model closely resembles the Bertrand game. Hence, the first stage is Stackelberg-solvable in the sense of d'Aspremont and GérardVaret (1980) and generates two asymmetric equilibria in pure strategies and consequently the mixed strategy equilibrium also becomes relevant.

The above Proposition has an obvious implication:

Corollary 3 If $\beta=1 / \sqrt{1-\sigma^{2}}$, the issue of choosing roles is immaterial.

This happens because reaction functions are orthogonal and therefore $\pi^{F}=\pi^{N}=\pi^{L}$ and the Nash and Stackelberg outcomes coincide.

To compute the equilibrium in mixed strategies at the pre-play stage, define by $\mathfrak{p}_{i}$ the probability that player $i$ attaches to $t_{1}$, so that the probability attached to $t_{2}$ is $1-\mathfrak{p}_{i}$. Player $i$ must set $\mathfrak{p}_{i}$ so as to make player $j$ indifferent between the two pure strategies. That is, payoffs

$$
\begin{aligned}
\pi_{j}\left(t_{1}, \mathfrak{p}_{i}\right) & =\mathfrak{p}_{i} \pi^{N}+\left(1-\mathfrak{p}_{i}\right) \pi^{L} \\
\pi_{j}\left(t_{2}, \mathfrak{p}_{i}\right) & =\mathfrak{p}_{i} \pi^{F}+\left(1-\mathfrak{p}_{i}\right) \pi^{N}
\end{aligned}
$$

must coincide. This happens at

$$
\widehat{\mathfrak{p}}_{i}=\frac{\pi^{L}-\pi^{N}}{\pi^{L}+\pi^{F}-2 \pi^{N}}
$$


with

$$
\pi_{j}\left(t_{1}, \mathfrak{p}_{i}\right) \gtreqless \pi_{j}\left(t_{2}, \mathfrak{p}_{i}\right) \forall \mathfrak{p}_{i} \gtreqless \widehat{\mathfrak{p}}_{i}
$$

Moreover, $\lim _{\beta \rightarrow 1 / \sqrt{1-\sigma^{2}}} \widehat{\mathfrak{p}}_{i}=0$,

$$
\begin{gathered}
\widehat{\mathfrak{p}}_{i} \leq 0 \forall \beta \in\left[0, \frac{1}{\sqrt{1-\sigma^{2}}}\right] \\
\widehat{\mathfrak{p}}_{i}>0 \forall \beta>\frac{1}{\sqrt{1-\sigma^{2}}}
\end{gathered}
$$

and

$$
\begin{gathered}
\lim _{s \rightarrow 1} \widehat{\mathfrak{p}}_{i}=\frac{(3+2 \beta)(5+2 \beta)}{[5+2 \beta(5+2 \beta)]^{2}} \\
\lim _{\beta \rightarrow \infty}\left(\lim _{s \rightarrow 1} \widehat{\mathfrak{p}}_{i}\right)=0 .
\end{gathered}
$$

As a result, $\widehat{\mathfrak{p}}_{i} \in(0,1)$ for all $\beta \in\left(1 / \sqrt{1-\sigma^{2}}, \infty\right)$. For any $\beta \in\left(1 / \sqrt{1-\sigma^{2}}, \infty\right)$, the map of best replies $\mathfrak{p}_{i}^{*}\left(\mathfrak{p}_{j}\right)$ in the space of probabilities $\left(\mathfrak{p}_{i}, \mathfrak{p}_{j}\right)$ looks like in Figure 2. This illustrates that:

- if $i$ chooses any $\mathfrak{p}_{i} \in(0,1)$, the best reply of $j$ is $\widehat{\mathfrak{p}}_{j}$;

- if $\mathfrak{p}_{i}=0$, the best reply of $j$ is any $\mathfrak{p}_{j} \in\left(0, \widehat{\mathfrak{p}}_{j}\right)$;

- if $\mathfrak{p}_{i}=1$, the best reply of $j$ is any $\mathfrak{p}_{j} \in\left(\widehat{\mathfrak{p}}_{j}, 1\right)$. 


\section{Figure 2}

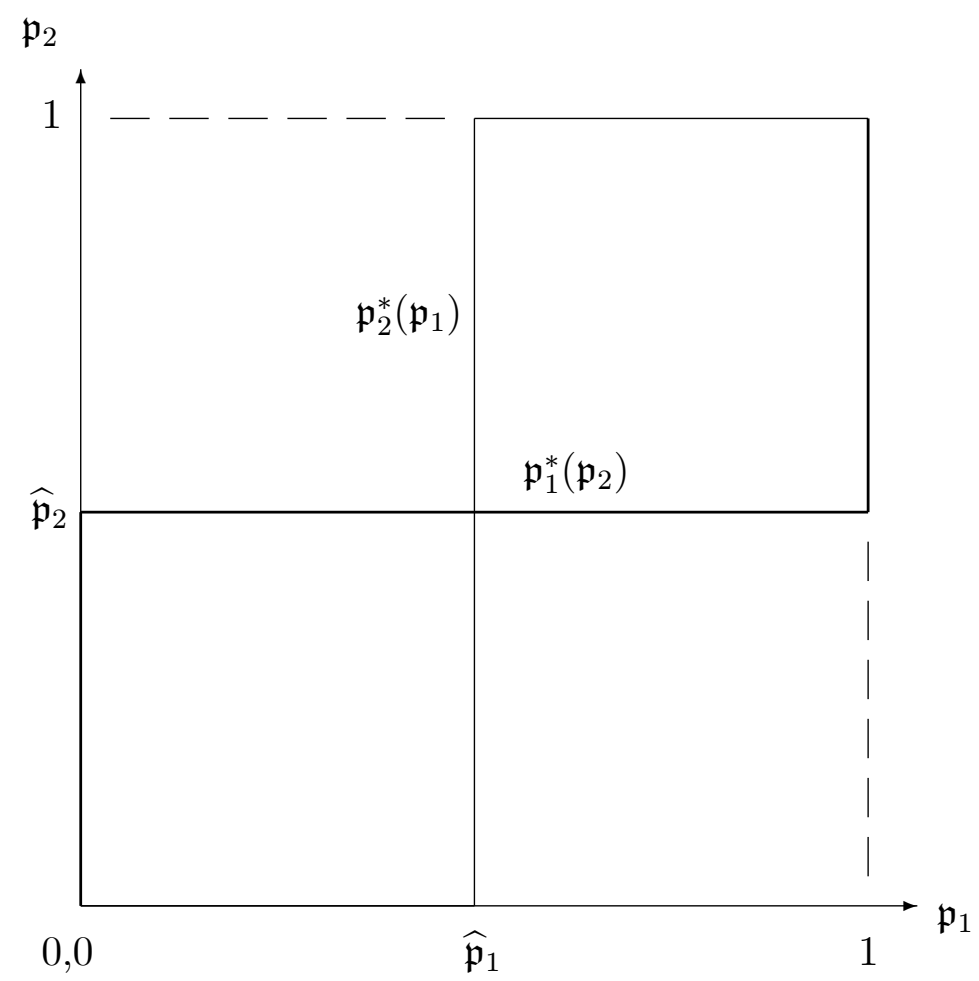

If instead $\beta \in\left[0,1 / \sqrt{1-\sigma^{2}}\right]$, then $\widehat{\mathfrak{p}}_{i} \in[-2 / 5,0]$ and the map of best replies is as in Figure 3. That is, as soon as $\beta$ is low enough as to make best replies orthogonal or downward sloping, firms select to play as early as possible and the Nash equilibrium results. 


\section{Figure 3}

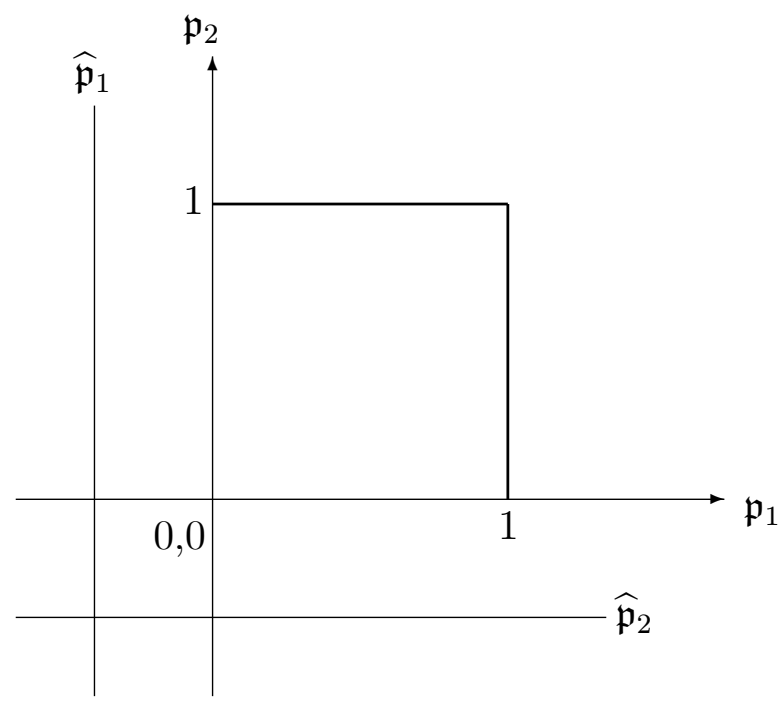

We can then claim the following:

Proposition 4 For all $\beta \in\left[0,1 / \sqrt{1-\sigma^{2}}\right]$, the mixed strategy Nash equilibrium collapses into the dominant strategy one, with $\mathfrak{p}_{i}^{*}=\mathfrak{p}_{j}^{*}=1$. For all $\beta \in\left(1 / \sqrt{1-\sigma^{2}}, \infty\right)$, the mixed strategy equilibrium is $\left(\widehat{\mathfrak{p}}_{i}, \widehat{\mathfrak{p}}_{j}\right)$.

Note that, in the limit, as product differentiation vanishes, $\lim _{\sigma \rightarrow 1}\left(1 / \sqrt{1-\sigma^{2}}\right)=$ $\infty$ and therefore firms are forced to select $\left(t_{1}, t_{1}\right)$, playing the dominant strategy equilibrium under strategic substitutability.

\section{Concluding remarks}

We have illustrated the endogenous timing of moves in a differentiated duopoly where firms compete in affine supply functions by choosing their intercepts 
as in Menezes and Quiggin (2012). To do so, we have relied upon the concept of extended game with observable delay (Hamilton and Slutsky, 1990). Since best replies are downward (upward) sloping if the common slope of supply functions is sufficiently low (high), simultaneous (sequential) play is part of the subgame perfect equilibrium strategy profile when best replies are negatively (positively) sloped. Hence, while Menezes and Quiggin (2012) use affine supply functions to reproduce Cournot and Bertrand Nash equilibria as limit cases, here we have shown that affine supply functions can also be used to reconstruct the same endogenous timing arising under quantity and price competition, as in Hamilton and Slutsky (1990). 


\section{References}

[1] Akgün, U. (2004), "Mergers with Supply Functions", Journal of Industrial Economics, 52, 535-46.

[2] Boyer, M. and M. Moreaux (1987), "On Stackelberg Equilibria with Differentiated Products: The Critical Role of the Strategy Space”, Journal of Industrial Economics, 36, 217-30.

[3] Bulow, J.I., J.D. Geanakoplos and P.D. Klemperer (1985), "Multimarket Oligopoly: Strategic Substitutes and Complements", Journal of Political Economy, 93, 488-511.

[4] Ciarreta, A. and C. Gutierrez-Hita (2006), "Supply Function vs Quantity Competition in Supergames", International Journal of Industrial Organization, 24, 773-83.

[5] d'Aspremont, C. and L.-A. Gérard-Varet (1980), "Stackelberg-solvable Games and Pre-play Communication", Journal of Economic Theory, 23, 201-17.

[6] Dowrick, S. (1986), "von Stackelberg and Cournot Duopoly: Choosing Roles", RAND Journal of Economics, 17, 251-60.

[7] Gal-Or, E. (1985), "First Mover and Second Mover Advantages", International Economic Review, 26, 649-53.

[8] Hamilton, J. and S. Slutsky (1990), "Endogenous Timing in Duopoly Games: Stackelberg or Cournot Equilibria", Games and Economic Behavior, 2, 29-46.

[9] Klemperer, P.D. and M.A. Meyer (1989), "Supply Function Equilibria in Oligopoly under Uncertainty", Econometrica, 57, 1243-77. 
[10] Menezes, F.M. and J. Quiggin (2012), "More Competitors or More Competition? Market Concentration and the Intensity of Competition", Economics Letters, 117, 712-14.

[11] Robson, A.J. (1990), "Duopoly with Endogenous Strategic Timing: Stackelberg Regained", International Economic Review, 31, 263-74.

[12] Singh, N. and X. Vives (1984), "Price and Quantity Competition in a Differentiated Duopoly", RAND Journal of Economics, 15, 546-54.

[13] Spence, A.M. (1976), "Product Differentiation and Welfare", American Economic Review, 66, 407-14.

[14] Vives, X. (2011), "Strategic Supply Function Competition with Private Information", Econometrica, 79, 1919-66. 


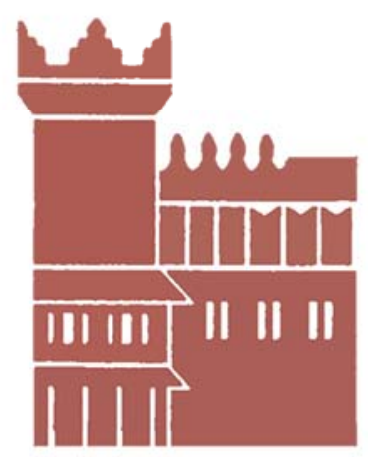

Alma Mater Studiorum - Università di Bologna DEPARTMENT OF ECONOMICS

Strada Maggiore 45

40125 Bologna - Italy

Tel. +39051 2092604

Fax +390512092664

http://www.dse.unibo.it 